\title{
Panorama mondial des réacteurs nucléaires de troisième génération
}

\section{Editorial}

Par Françoise TERNON-MORIN, EDF, Présidente 2013 de la ST7

et Joël GUIDEZ, CEA, Président 2014 de la ST7

Les journées des 5 et 6 décembre 2013, organisées par les Sections techniques ST 7 (Technologie et Exploitation des Réacteurs) et ST 8 “Economie et Stratégie Energétique”, étaient consacrées aux "Réacteurs nucléaires de demain". Ces journées débutaient par un panorama mondial des réacteurs nucléaires de troisième génération qui seront très clairement les principaux réacteurs du vingt-etunième siècle et qui font l'objet de ce premier numéro 2014 de la RGN.

\section{Q Qu'est-ce qu'un réacteur nucléaire de troisième génération?}

Les réacteurs de troisième génération (Gen3) sont des réacteurs évolutionnaires intégrant le retour d'expérience des réacteurs de deuxième génération (Gen2) conçus dans les années 70 et des principes physiques éprouvés sans saut technologique.

La nouvelle conception de ces réacteurs vise d'abord à renforcer leur sûreté sur trois points principaux :

- Un risque d'accident grave avec fusion du cœur abaissé à une probabilité de $10^{-5}$ par réacteur et par an, toutes causes confondues ;

- Une prise en compte des accidents graves dès la conception avec garantie du bon comportement du confinement, des possibilités de mitigation et de limitation correspondante des rejets ;

- Une meilleure protection contre les agressions externes (chutes d'avion, séisme, inondation).

Elle vise ensuite une amélioration de l'acceptabilité par le public grâce à ses dispositions basées sur les retours d'expérience successifs des accidents de Three Mile Island en 1979, de Tchernobyl en 1986 et de Fukushima en 2011, en démontrant une limitation drastique de l'impact radiologique des accidents, y compris d'un accident grave avec fusion du cœur et par conséquent de la zone de plan d'urgence.
Ces réacteurs prennent aussi en compte des objectifs d'amélioration des performances économiques :

- des objectifs de performance opérationnelle élevés, basés sur l'utilisation de techniques éprouvées et fiabilisées (Disponibilité > $90 \%$ ),

- Des arrêts pour rechargement courts (spécification EUR révision $\mathrm{D}: 16$ jours),

- Une amélioration des possibilités de maintenance en fonctionnement,

- Une durée de vie de l'installation d'au moins 60 ans.

Les réacteurs Gen3 se distinguent de la génération 4 par le fait qu'ils ne répondent pas en général à l'objectif de développement durable vis-à-vis des ressources naturelles, notamment de l'utilisation du combustible (Uranium naturel).

A noter que les 440 réacteurs nucléaires en fonctionnement dans le monde sont très majoritairement des réacteurs à eau, avec environ $2 / 3$ de technologie à eau pressurisée (PWR) et un tiers de technologie bouillante (BWR).

Vers une harmonisation des règles de sûreté et des exigences de conception ?

Si les principes généraux sont posés, les approches techniques pour y répondre dépendent cependant des constructeurs, par exemple du choix de systèmes passifs et/ou actifs ou du choix des systèmes de mitigation des accidents graves. 
Cependant, on observe, dès les années 90 , une volonté forte d'harmonisation internationale de ces objectifs de sûreté, et des exigences correspondantes de conception.

Cette volonté a été accélérée par la prise en compte du retour d'expérience de l'accident de Fukushima qui a conduit à une convergence et à la publication des documents correspondants. On peut citer par exemple les documents AIEA (Specific Safety Requirements SSR2.1,

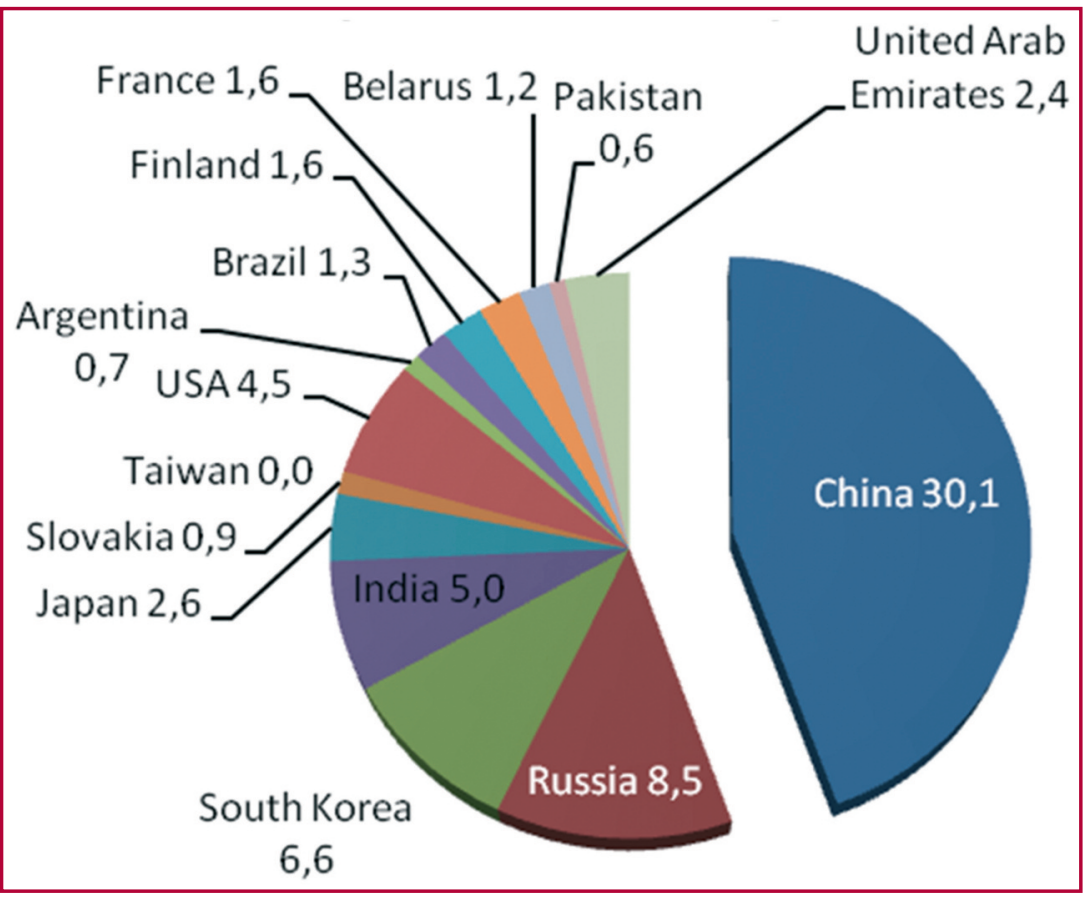

Fig. 1 - Puissance des réacteurs en construction dans le monde (en GW par pays)

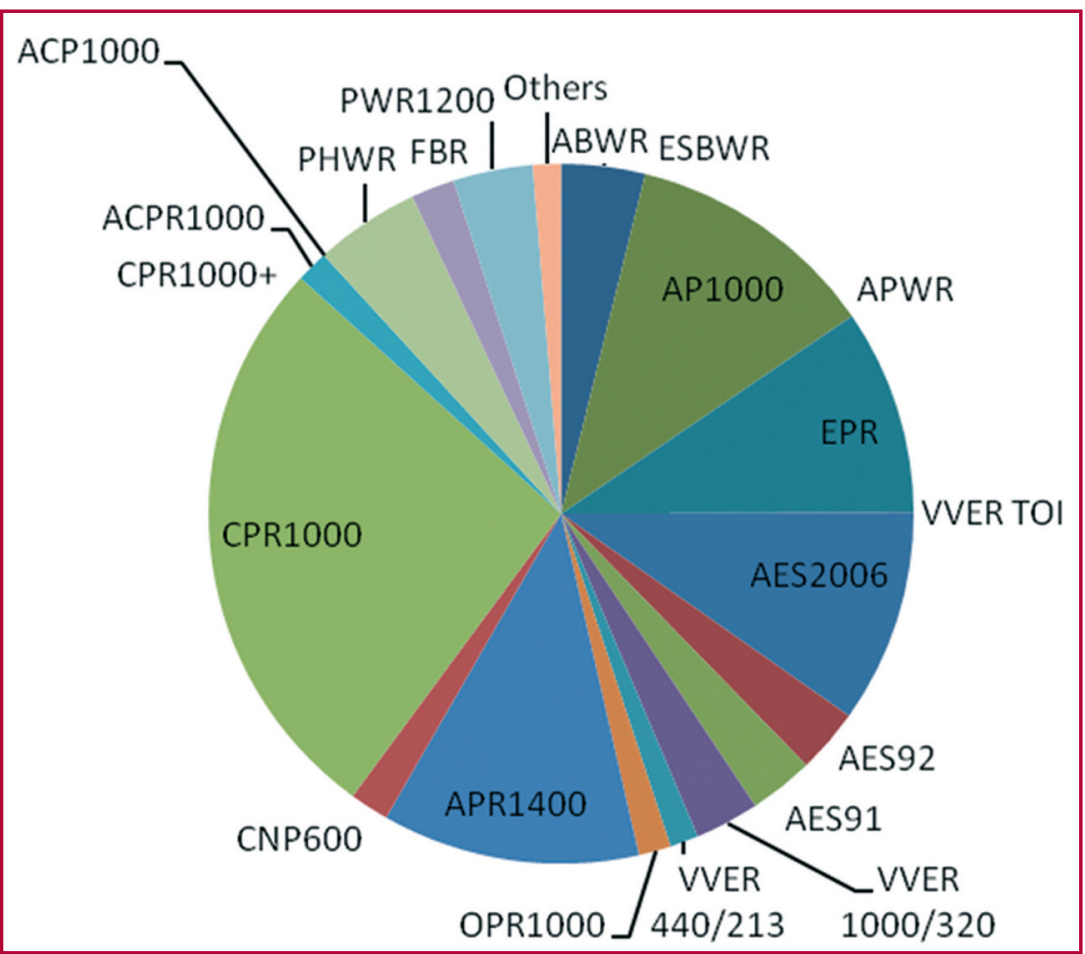

Fig. 2 - Types de design des réacteurs en construction dans le monde
2012), WENRA (Safety of New NPP Designs, Mars 2013), EUR (Révision D, octobre 2012).

On notera en particulier dans les évolutions des règles de sûreté :

- La prise en compte des événements externes très rares et des défaillances de cause commune,

- La demande d'indépendance des systèmes impliqués dans différents niveaux de défense en profondeur,

- Une meilleure considération du stockage de combustible usé,

- L'augmentation des autonomies,

- La disponibilité d'équipements mobiles,

- En France la mise en place de forces d'intervention rapide (FARN).

\section{Bilan des réacteurs en cons- truction dans le monde}

En 2013, 69 réacteurs (67 GWe) sont en construction dans le monde, dont les deux tiers en Chine et en Russie. En dehors de deux réacteurs rapides sodium (PFBR en Inde et BN800 en Russie), ainsi que de deux HTR en construction en Chine, les réacteurs en construction sont principalement des réacteurs à eau pressurisée de type PWR (REP) et quelques réacteurs bouillants de type BWR (REB).

Concernant les différences entre BWR et PWR, on trouvera dans ce numéro de la RGN un article comparant ces deux filières en donnant les avantages/inconvénients de chacune d'elles, ainsi qu'une mise en perspective de leur développement.

Quelques réacteurs de deuxième génération sont actuellement en construction (essentiellement en Chine) mais le standard Gen3 semble actuellement retenu pour toute nouvelle construction.

\section{- L'offre française}

Actuellement l'offre française est essentiellement portée par I'EPR d'AREVA.

Les caractéristiques de ce réacteur correspondent aux critères des réacteurs de troisième génération (figure 3 ). Plusieurs exemplaires sont actuellement, en construction en Finlande, en France sur le site de Flamanville (figure 4), et en Chine à Taishan. Deux sont en projet en Angleterre sur le site d'Hinkley Point.

Plusieurs présentations du réacteur EPR ont été effectuées dans le numéro $5 / 2013$ de la RGN, consacré à l'exportation, et aucune présentation n'a donc été reprise dans ce numéro. 


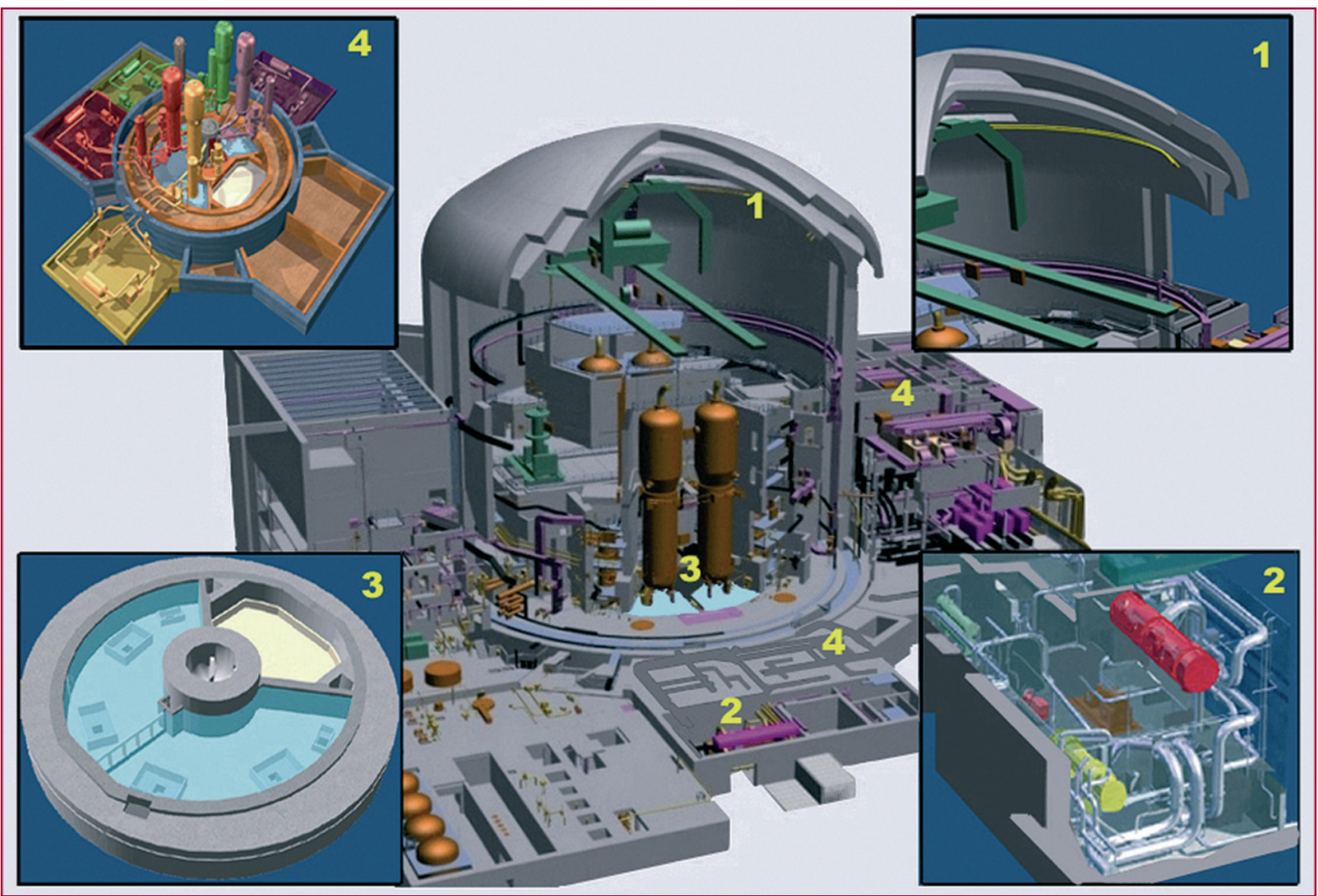

Fig. 3 - Vue du réacteur EPR d'AREVA et ses principales caractéristiques

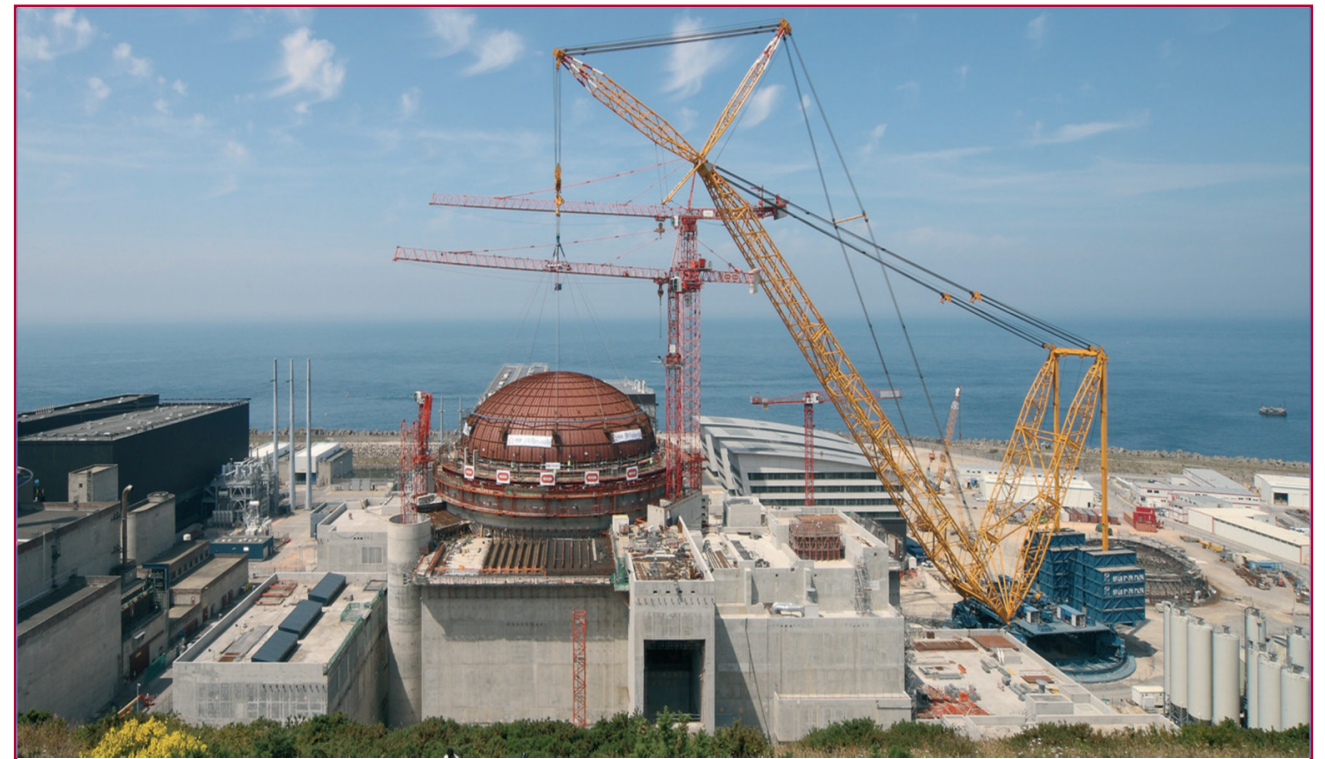

Fig. 4 - Pose du dôme d'EPR Flamanville 3 en juillet 2013
C'est un PWR évolutionnaire de $1100 \mathrm{MWe}$ à 3 boucles primaires dont les principales caractéristiques sont :

- 3 trains de sûreté mécaniques et électriques $(3+1$ pour certains systèmes support),

- des accumulateurs avancés MHI (systèmes passifs),

- Une enceinte monocoque (prise en compte de chute d'avion commercial),

- Une approche accident grave, avec core catcher primaire.

On trouvera une description détaillée du concept de I'ATMEA1 dans l'article correspondant du présent numéro.

Mais le catalogue de l'offre française comprend aussi un réacteur PWR ATMEA1 conçu par la Joint Venture ATMEA (50 \% AREVA-50 \% Mitsubishi) et un BWR KERENA conçu sur la base de l'expérience des BWR allemands.

ATMEA 1 s'inspire des technologies (EPR et APWR) des deux entreprises co-fondatrices.
Le réacteur KERENA est un réacteur BWR conçu sur la base de l'expérience de fonctionnement des réacteurs bouillants allemands. II présente en particulier un certain nombre de dispositifs passifs innovants. On trouvera une description détaillée de ce réacteur dans l'article correspondant de ce numéro. 


\section{- L'offre internationale}

Actuellement l'offre internationale en réacteurs de troisième génération comporte des réacteurs russes, japonais, américains, coréens et chinois, les Chinois étant les derniers acteurs arrivés sur ce marché. Les principales offres sur le marché sont les suivantes :

\section{1- Technologie PWR :}

- AP1000 de Westinghouse (Groupe Toshiba),

- AES 91/92/2006/VVER TOI de JSC AEP \& SPbAEP (Corporation Rosatom),

- APR1400 de KEPCO,

- APWR de MHI,

- ATMEA 1 d'ATMEA (co-entreprise AREVA + MHI),

- réacteurs de conception chinoise : ACP 1000 (CNNC), ACPR1000+ (CGN), CAP 1400 (SNPTC).
L'article de ce numéro sur les réacteurs russes donne la description détaillée de l'ensemble de cette offre.

\section{- APR1400 de KEPCO}

C'est un réacteur PWR de 1450 MWe conçu par KEPCO. Plusieurs versions sont disponibles, dont une version européenne à 4 trains, avec un récupérateur corium et une enceinte à double coque (ce qui n'est pas le cas sur la version coréenne d'origine). La salle de commande et le contrôle commande sont fournis par Westinghouse.

Plusieurs de ces réacteurs sont en construction en Corée (Shin Kori) et aux Emirats Arabes Unis (Barakah) (figure 5). - APWR de MHI

\section{2- Technologie BWR :}

- ABWR de GE-Hitachi / Toshiba,

- ESBWR de GE-Hitachi.

On notera que les éléments de comparaison entre PWR et BWR font l'objet d'un article spécifique dans ce numéro.

\section{- AP1000 de Toshiba-Westinghouse}

C'est un réacteur PWR "passif" de 1130 MWe dérivé de l'AP 600 certifié aux USA en 1998. II se caractérise principalement par un système d'injection de sécurité passif, par une rétention du cœur fondu en cuve (sans récupérateur de corium) et par le recours à une technique de construction modulaire. Huit réacteurs sont en construction : 4 aux USA (Vogtle, VC Summer) et 4 en Chine (Sanmen, Haiyang).

II fait l'objet de deux articles dans ce numéro.

\section{- Réacteurs russes}

La Russie a relancé son programme nucléaire avec de nouveaux réacteurs de troisième génération :

\section{- AES 2006}

Cette centrale de 1200 MWe dérive de la conception des AES 91 et 92 . Deux centrales sont en construction en Russie : à Novoronezh (1\&2) et Leningrad (1\&2). Les réacteurs y disposent de systèmes passifs d'évacuation de puissance résiduelle et de récupérateurs de corium.

\section{- VVER TOI}

Ce nouveau design est une évolution/ optimisation du réacteur de l'AES 2006 (version AEP Moscou) caractérisée par : - Une puissance légèrement augmentée,

- Un coût de fabrication optimisé (-20\%) et un planning de construction plus court (40 mois hors Tête de série),

- Une amélioration des caractéristiques d'exploitation.

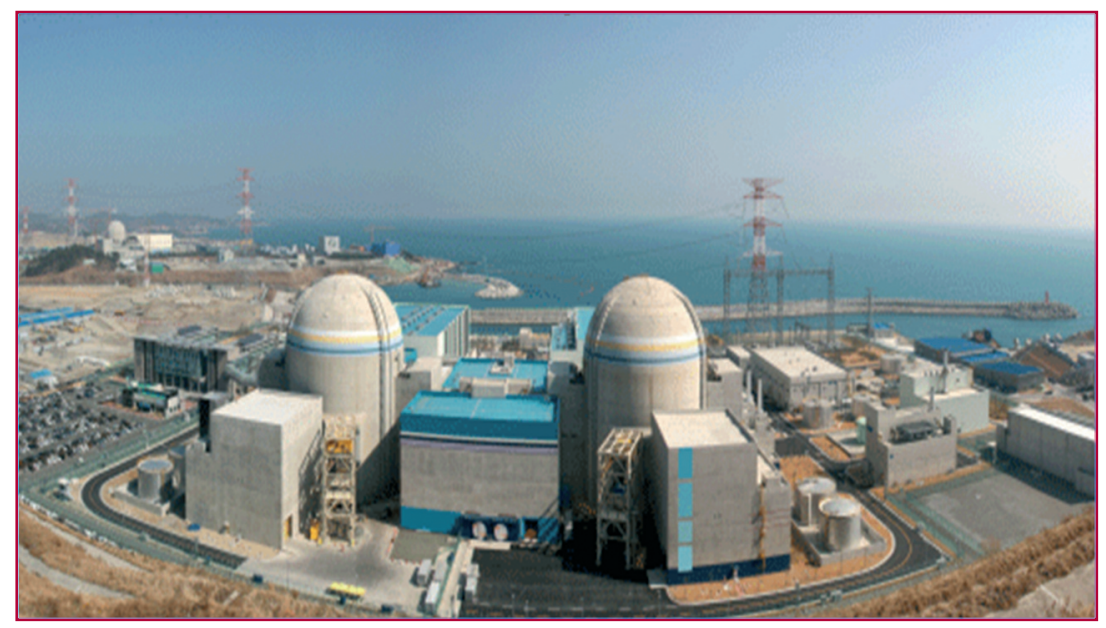

Fig. 5 - Vue des réacteurs APR 1400 en construction à Shin Kori

C'est un réacteur PWR développé par $\mathrm{MHI}$ dont la puissance varie entre 1550 MWe (version japonaise) et $1700 \mathrm{MWe}$ (version US ou Europe). C'est un PWR à systèmes actifs de sauvegarde, avec récupérateur de corium et enceinte monocoque renforcée. 


\section{- Réacteurs chinois}

C'est la Chine qui a le plus grand programme de construction de réacteurs nucléaires dans le monde (avec 31 réacteurs)

On y trouve une grande variété de réacteurs : des WER russes, des AP1000 de Westinghouse, des EPR français, des réacteurs "sinisés" dérivés des réacteurs de deuxième génération CP100 mais aussi deux HTR. Pour toutes ces versions, des transferts de technologie sont en cours et une "sinisation" sera rapidement effectuée pour ce pays qui affiche déjà des volontés à l'export.

\section{- ABWR de GE-Hitachi/Toshiba}

L'ABWR (Advanced Boiling Water Reactor) est un réacteur à eau bouillante de $1350 \mathrm{MWe}$, développé à la fin des années 80 par un consortium impulsé par TEPCO et piloté par General Electric avec Hitachi, Toshiba, Ansaldo (ASEA Atom au début). Une version de 1600 MWe a été conçue pour l'Europe (EU-ABWR). L'enceinte de confinement est en béton armé, avec peau métallique interne à l'intérieur d'un bâtiment réacteur non conçu à l'origine pour résister à la chute d'un avion gros porteur. L'architecture d'ensemble intègre des améliorations déjà mises en œuvre par ASEA ou Siemens dans les années 70 et 80 : pompes de recirculation internes à rotors noyés et mécanismes de barres à doubles actionneurs (piston et moteur-vis). Les systèmes de sauvegarde ( 3 trains mécaniques et électriques) sont actifs et il dispose d'un récupérateur de corium (figure 7 ).

Sa description est effectuée plus en détail dans l'article correspondant de ce numéro.

\section{- ESBWR de GE-Hitachi}

C'est un réacteur bouillant "passif" de 1530 MWe dérivé de I'ABWR. II a été développé conjointement par General Electric et Hitachi. Les pompes de circulation du primaire ont été supprimées pour être remplacées par la convection naturelle.

L'enceinte n'était pas conçue initialement pour résister aux chutes d'avion, et le réacteur dispose d'un récupérateur corium avec refroidissement passif (figure 8).

\section{- Les perspectives mondiales}

Les prévisions ont été revues à la baisse principalement du fait :

- d'un contexte de crise mondiale persistante rendant difficiles pour les acteurs occidentaux les conditions de financement,

- de l'impact de l'accident de Fukushima,

- de l'essor et du bas prix des gaz de schiste et de la baisse consécutive des prix du charbon,
- de la possibilité et l'intérêt économique de prolongation de la durée de vie des installations existantes,

- de la durée d'acquisition par les nouveaux entrants du cadre nécessaire à la réalisation d'un projet de construction (réglementations, autorité de sûreté,...) et des compétences adéquates.

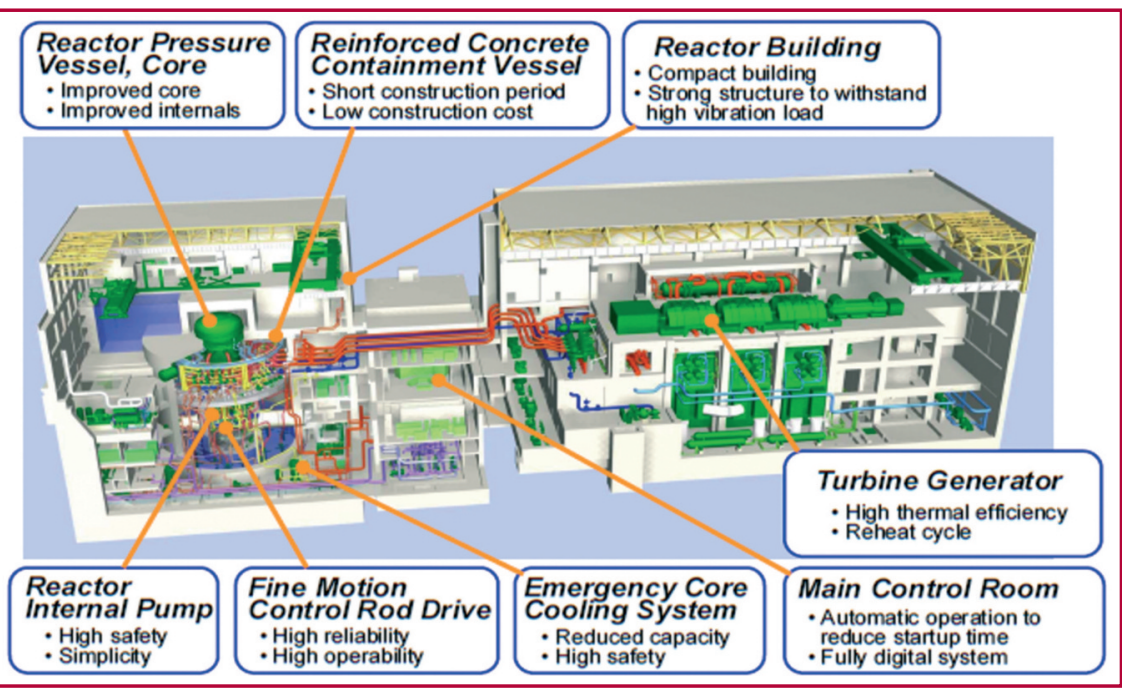

Fig. 7 - Vue du réacteur ABWR

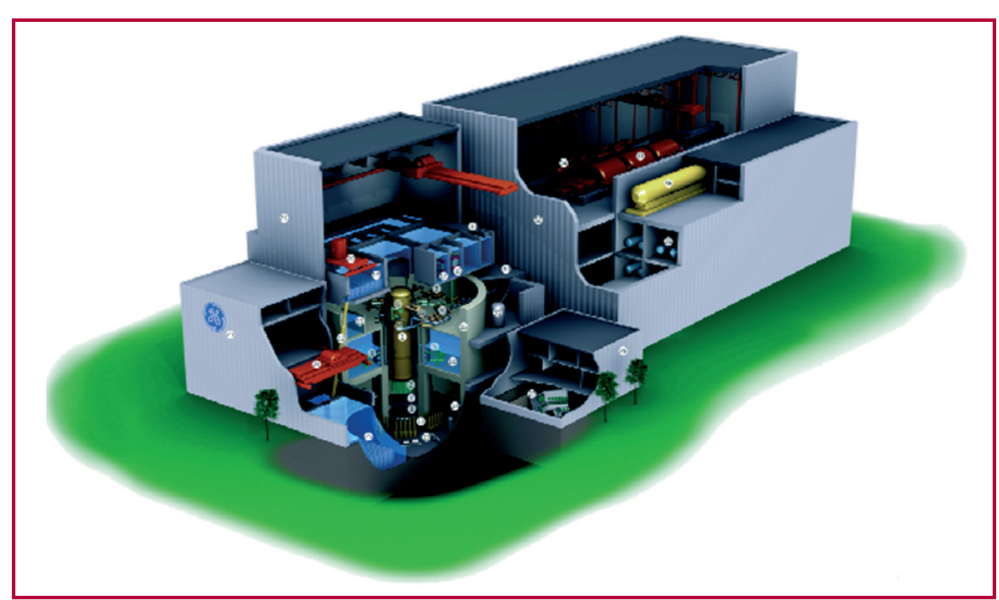

Fig. 8 - Vue du réacteur ESBWR de GE-Hitachi

\section{Conclusion}

De nombreux designs de Génération 3 sont disponibles sur le marché mondial, avec des objectifs de conception accrus en matière de sûreté et de performance en exploitation.

Certains designs évolutionnaires disposent d'innovations telles que systèmes passifs et recours croissant à une modularisation pour réduire le temps de construction.

Certains précurseurs sont déjà en exploitation (premiers ABWR, AES 91-92) alors que la plupart des autres têtes de série GEN3 sont à un stade avancé de construction (EPR, AP1000, APR1400, AES 2006)

Les perspectives de développement du nucléaire à l'international ont été revues à la baisse suite à l'accident de Fukushima mais elles restent importantes pour ces réacteurs qui seront la base des réacteurs nucléaires du $21^{\text {e }}$ siècle. 